\section{PREVALÊNCIA DE INCONTINÊNCIA URINÁRIA E FATORES ASSOCIADOS EM MULHERES NO CLIMATÉRIO EM UMA UNIDADE DE ATENÇÃO PRIMÁRIA À SAÚDE}

\author{
Prevalence of urinary incontinence and associated factors in \\ climacteric women of a primary health care unit
Prevalencia de incontinencia urinaria y los factores asociados en mujeres en el climaterio de una unidad de atención primaria a la salud

\section{RESUMO}

Objetivo: Estimar a prevalência de incontinência urinária (IU) em mulheres no climatério e investigar os fatores associados. Métodos: Trata-se de um estudo quantitativo, do tipo transversal e analítico com 233 mulheres no climatério, na faixa etária de 40 a 65 anos, atendidas em uma Unidade de Atenção Primária a Saúde (UAPS), desenvolvido no período de dezembro de 2014 a junho de 2015. Coletaram-se os dados sociodemográficos, comorbidades, clínicos (obstétricos e ginecológicos) e perfil urinário. Utilizou-se o programa SPSS 19.0 e o teste de qui-quadrado para analisar associação entre as variáveis. Adotouse nível de significância de 5\%. Resultados: A queixa de IU esteve presente em 41,2\% $(\mathrm{n}=96)$ das mulheres. Verificou-se maior prevalência na faixa etária de 40-45 anos com $36,5 \%(n=35)(p=0,05)$, cor parda com $21,9 \%(n=21)(p=0,002)$ e escolaridade baixa (até $1^{\circ}$ ciclo fundamental completo) com 39,6\% ( $\left.=38\right)(\mathrm{p}=0,627)$. Quanto à comorbidades autorreferidas, 38,5\% $(\mathrm{n}=37)$ das mulheres incontinentes apresentaram hipertensão arterial sistêmica $(\mathrm{p}=0,96), 17,7 \%(\mathrm{n}=17)$ diabetes $(\mathrm{p}=0,982)$ e $28,1 \%(\mathrm{n}=27)$ constipação intestinal $(\mathrm{p}=0,101)$. Sobre as características clínicas, $41,7 \%(\mathrm{n}=35)$ tiveram apenas partos do tipo vaginal $(\mathrm{p}=0,087)$ e $53,1 \%(\mathrm{n}=51)$ estavam na menopausa $(\mathrm{p}=0,113)$. Verificou-se a IU de esforço em 85,4\% (n=82) das mulheres. Conclusão: Encontrou-se elevada prevalência de incontinência urinária em mulheres climatéricas, destacando maior ocorrência nas mais jovens e de cor parda.

Descritores: Incontinência Urinária; Prevalência; Climatério.

\section{ABSTRACT}

Objective: To estimate the prevalence of urinary incontinence (UI) and investigate the associated factors. Methods: This is a quantitative, cross-sectional and analytical study with 233 women going through climacteric, in the age range of 40-65 years, seen in a Primary Health Care Unit (PHU), developed in the period from December 2014 to June 2015. Sociodemographic and clinical (obstetrical and gynecological) data, comorbidities and urinary profile were assessed. The software SPSS 19.0 and the chi-square test were employed to evaluate the association between the variables. The significance level of 5\% was adopted. Results: Complaint about UI was present in $41.2 \%(n=96)$ of women. Higher prevalence was observed in the age group of 40-45 years with $36.5 \%(n=35)(p<0.05)$, brown-skinned with $21.9 \%(n=21)(p=0.002)$, and low level of education (up to complete Junior High) with 39.6\% $(n=38)(p=0.627)$. As for the self-reported comorbidities, $38.5 \%$ $(n=37)$ of the incontinent women presented systemic arterial hypertension $(p=0.96), 17.7 \%$ $(n=17)$ had diabetes $(p=0.982)$, and 28.1\% $(n=27)$, constipation $(p=0.101)$. Regarding the clinical characteristics, $41.7 \%(n=35)$ had only vaginal delivery $(p=0.087)$ and $53.1 \%$ $(n=51)$ were going through menopause $(p=0.113)$. The stress urinary incontinence (SUI) was found in $85.4 \%(n=82)$ of the women. Conclusion: A high prevalence of urinary incontinence in climacteric women was found, emphasizing higher frequency among the younger, brownskinned women.

Descriptors: Urinary Incontinence; Prevalence; Climacteric.
Artigo Original

Ticiana Mesquita de Oliveira ${ }^{(1)}$ Flávia Muniz Lelis Valdez ${ }^{(1)}$ Karla Erica dos Santos Lima ${ }^{(1)}$ Milena Sampaio Magalhães ${ }^{(1)}$ Ana Paula Vasconcellos Abdon ${ }^{(1)}$ Ilana Nogueira Bezerra ${ }^{(1)}$

1) Universidade de Fortaleza - UNIFOR Fortaleza (CE) - Brasil
Recebido em: 28/11/2015 Revisado em: 10/12/2015 Aceito em: 29/12/2015 


\section{RESUMEN}

Objetivo: Estimar la prevalencia de incontinencia urinaria (IU) en mujeres en el climaterio y investigar los factores asociados. Métodos: Se trata de un estudio cuantitativo del tipo transversal $y$ analítico con 233 mujeres en el climaterio, en la franja de edad entre 40 y 65 años asistidas en la Unidad de Atención a la Salud (UAPS) desarrollado en el periodo de diciembre de 2014 a junio de 2015. Se recogieron datos sociodemográficos, de comorbidades, clínicos (obstétricos y ginecológicos) y del perfil urinario. Se utilizo el programa SPSS 19.0 y la prueba chi-cuadrado para analizar la asociación entre las variables. Se adoptó el nivel de significancia del 5\%. Resultados: La queja de IU estuvo presente en el 41,2\% (n=96) de las mujeres. Se verificó mayor prevalencia en la franja de edad entre 40-45 años en el 36,5\% $(n=35)(p=0,05)$, color pardo en el $21,9 \%(n=21)(p=0,002)$ y baja escolaridad (hasta el $1^{\circ}$ ciclo fundamental completo) en el 39,6\% ( $\left.n=38\right)$ $(p=0,627)$. Respecto las comorbidades autorreferidas, el $38,5 \%$ $(n=37)$ de las mujeres con incontinencia presentaron hipertensión arterial sistémica $(p=0,96)$, el 17,7\% $(n=17)$ diabetes $(p=0,982)$ $y$ el 28,1\% $(n=27)$ constipación intestinal $(p=0,101)$. Sobre las características clínicas, el 41,7\% (n=35) tuvieron solamente partos del tipo vaginal $(p=0,087)$ y el 53,1\% ( $n=51)$ estaban en la menopausia $(p=0,113)$. Se verificó la IU de esfuerzo en el $85,4 \%(n=82)$ de las mujeres. Conclusión: Se encontró elevada prevalencia de IU en mujeres en el climaterio destacando mayor ocurrencia en las jóvenes de color pardo.

Descriptores: Incontinencia Urinaria; Prevalencia; Climaterio.

\section{INTRODUÇÃO}

O climatério e definido como uma fase biológica da vida e não um processo patológico, que compreende a transição entre o período reprodutivo (menacme) e o não reprodutivo (senectude) ${ }^{(1)}$. Este período da vida da mulher, decorrente do progressivo e rápido esgotamento dos folículos ovarianos, caracteriza-se pela queda progressiva dos hormônios femininos e na instalação de sérios agravos, como osteoporose, doença cardiovascular e incontinência urinária (IU) $)^{(2)}$.

Dentre as doenças mais prevalentes na população feminina, estima-se que a incontinência urinária (IU) atinja mais de 50 milhões de pessoas em todo o mundo, acometendo aproximadamente $25 \%$ de mulheres jovens ( 14 e 21 anos de idade), $44 \%$ a 57\% na pós-menopausa (40 e 60 anos) e $75 \%$ de mulheres idosas ${ }^{(3)}$. No Brasil, cerca de 11 a $23 \%$ das mulheres apresentam este distúrbio ${ }^{(4)}$.

A IU é definida pela International Continence Society (ICS), como a queixa de qualquer perda involuntária de urina $^{(5)}$, podendo ser classificada em: incontinência urinária de esforço (IUE), incontinência urinária de urgência (IUU) e incontinência urinária mista $(\mathrm{IUM})^{(6)}$.
No entanto, acredita-se que essa prevalência esteja subestimada, uma vez que muitos casos não são diagnosticados e pelo fato da população acreditar que a IU seja uma condição normal, resultante do processo de envelhecimento ${ }^{(7)}$. Além disso, algumas mulheres mostramse relutantes em mencioná-lo aos profissionais de saúde ${ }^{(8)}$.

Estudos apontam que o período do climatério é visto como um dos responsáveis pelo surgimento da IU por ocorrer, neste período, uma diminuição do colágeno e da ação do estrogênio nas estruturas uroginecológicas, que levam a atrofia desta região e, consequente, diminuição da pressão de fechamento uretral ${ }^{(9)}$.

No entanto, a etiologia da incontinência urinária é multifatorial. Além do climatério, outros fatores estão associados à ocorrência de IU na população feminina, destacando-se fatores sociodemográficos, obstétricos, comportamentais e comorbidades ${ }^{(10)}$.

Baseado na escassez de estudos que relacione estes fatores com a incontinência urinária no período do climatério, em particular no estado do Ceará, esse trabalho objetivou estimar a prevalência de incontinência urinária (IU) em mulheres no climatério e investigar os fatores associados.

\section{MÉTODOS}

Trata-se de um estudo quantitativo, observacional, do tipo transversal e analítico com mulheres atendidas na Unidade de Atenção Primária à Saúde (UAPS), desenvolvido no período de dezembro de 2014 a junho de 2015.

Participaram deste estudo 274 mulheres na faixa etária de 40 a 65 , selecionadas de forma voluntária e que autorizaram sua participação por meio da assinatura do termo de consentimento livre e esclarecido. Porém, 41 mulheres foram retiradas do estudo devido ao não preenchimento correto dos instrumentos de coleta, totalizando uma amostra de 233 mulheres.

Os critérios de exclusão adotados foram mulheres grávidas, com diagnóstico de câncer, doenças renais, neurológicas, vasculares, alergia alimentar ou mulheres com alterações mentais/psicológicas que causassem dificuldade de expressar um raciocínio analítico quanto a perda urinária.

A coleta dos dados foi realizada na sala de espera da UAPS com duração média de 25 minutos. Aplicou-se um questionário, elaborado pelos pesquisadores, contendo os dados sociodemográficos (idade, estado civil, etnia, escolaridade e renda familiar), sobre comorbidades (hipertensão arterial sistêmica, diabetes mellitus e constipação), obstétricos e ginecológicos (numero de gestações, número de filhos, tipo de parto e menopausa) e perfil urinário (perda urinária, quantidade de urina, classificação de IU) e presença da incontinência urinária. 
Para avaliação de perdas urinárias, aplicou-se o Questionário Internacional de Incontinância [International Consultation on Incontinence Questionnaire - Short Form (ICIQSF)], que avalia o impacto da incontinência urinária sobre qualidade de vida e também qualifica a perda urinária. O ICIQ-SF traduzido e validado para a língua portuguesa é um instrumento composto por quatro questões que avaliam a frequência, a gravidade e o impacto da incontinência urinária, além de um conjunto de oito itens relacionados às causas ou situações de incontinência urinária vivenciadas ${ }^{(11)}$. Consideraram-se as mulheres incontinentes (com IU) quando o somatório do ICQ-SF foi igual ou superior a 3 pontos.

Utilizou-se o programa EpiInfo 7.0 para digitação dos dados com controle de qualidade na entrada dos dados para evitar falhas na amplitude e consistência dos dados. Posteriormente, os dados foram analisados pelo programa SPSS 19.0 usando estatística descritiva ou inferencial. Para verificar a relação entre as variáveis de exposição (sociodemográficas, comorbidades, obstétricas e ginecológicas) com a variável desfecho (presença de IU) utilizou-se o teste do qui-quadrado ( $\left.\chi^{2}\right)$. Adotou-se nível de significância de 5\%.

Esta pesquisa respeitou os padrões éticos e científicos estabelecidos pela Resolução no 466/12 do Conselho Nacional de Saúde/Ministério da Saúde sobre pesquisa envolvendo seres humanos, tendo sido aprovada pelo Comitê de Ética da Universidade de Fortaleza (parecer $n^{\circ}$. 520.906).

\section{RESULTADOS}

A amostra foi composta por 233 mulheres no climatério, das quais $41,2 \%(\mathrm{n}=96)$ apresentavam queixas de incontinência urinária. Verificou-se maior prevalência na faixa etária de $40-45$ anos com $36,5 \%(n=35)(\mathrm{p}=0,05)$,

Tabela I - Perfil sociodemográfico e comorbidades de acordo com a presença ou não de incontinência urinária. Fortaleza, CE, 2014-2015.

\begin{tabular}{|c|c|c|c|c|c|}
\hline \multirow{2}{*}{ Variáveis } & \multicolumn{2}{|c|}{ COM IU } & \multicolumn{2}{|c|}{ SEM IU } & \multirow{2}{*}{ Valor de $p$} \\
\hline & $n=96$ & $\%$ & $\mathrm{n}=\mathbf{1 3 7}$ & $\%$ & \\
\hline \multicolumn{6}{|l|}{ Idade } \\
\hline $40-45$ & 35 & 36,5 & 29 & 21,2 & \multirow[t]{5}{*}{$0,050^{*}$} \\
\hline $46-50$ & 25 & 26,0 & 32 & 23,4 & \\
\hline $51-55$ & 14 & 14,6 & 33 & 24,1 & \\
\hline $56-60$ & 12 & 12,5 & 27 & 19,7 & \\
\hline $61-65$ & 10 & 10,4 & 16 & 11,7 & \\
\hline \multicolumn{6}{|l|}{ Estado Civil } \\
\hline Solteiro & 28 & 29,2 & 43 & 31,4 & \multirow[t]{4}{*}{0,839} \\
\hline Casado & 54 & 56,3 & 70 & 51,1 & \\
\hline Divorciado & 8 & 8,3 & 12 & 8,8 & \\
\hline desquitado/separado & 6 & 6,3 & 12 & 8,8 & \\
\hline \multicolumn{6}{|l|}{ Raça } \\
\hline Branca & 21 & 21,9 & 38 & 27,7 & \multirow[t]{5}{*}{$0,002^{*}$} \\
\hline Preta & 28 & 29,2 & 12 & 8,8 & \\
\hline Amarela & 2 & 2,1 & 2 & 1,5 & \\
\hline Parda & 44 & 45,8 & 83 & 60,6 & \\
\hline Indígena & 1 & 1,0 & 2 & 1,5 & \\
\hline \multicolumn{6}{|l|}{ Escolaridade } \\
\hline Sem instrução $/ 1^{\circ}$ ciclo fundamental incompleto & 32 & 33,3 & 54 & 39,4 & \multirow{3}{*}{0,627} \\
\hline $1^{\circ}$ ciclo fundamental completo & 38 & 39,6 & 48 & 35,0 & \\
\hline $2^{\circ}$ ciclo fundamental completo ou mais & 26 & 27,1 & 35 & 25,5 & \\
\hline \multicolumn{6}{|l|}{ Renda familiar } \\
\hline Até $1 \mathrm{SM}$ & 45 & 46,9 & 70 & 51,1 & \multirow[t]{2}{*}{0,526} \\
\hline Maior que $1 \mathrm{SM}$ & 51 & 53,1 & 67 & 48,9 & \\
\hline \multicolumn{6}{|l|}{ Hipertensão arterial sistêmica (HAS) } \\
\hline Sim & 37 & 38,5 & 53 & 38,7 & \multirow[t]{2}{*}{0,916} \\
\hline Não & 59 & 61,5 & 84 & 61,3 & \\
\hline \multicolumn{6}{|l|}{ Diabetes Mellitus (DM) } \\
\hline $\operatorname{Sim}$ & 17 & 17,7 & 25 & 18,2 & \multirow[t]{2}{*}{0,982} \\
\hline Não & 79 & 82,3 & 112 & 81,8 & \\
\hline \multicolumn{6}{|l|}{ Constipação } \\
\hline $\operatorname{Sim}$ & 27 & 28,1 & 26 & 19,0 & \multirow[t]{2}{*}{0,101} \\
\hline Não & 69 & 71,9 & 111 & 81,0 & \\
\hline
\end{tabular}

$\mathrm{IU}=$ incontinência urinária; $\mathrm{SM}=$ Salário Mínimo. ${ }^{*} \mathrm{p}<0,05$. 
cor parda com $21,9 \%(\mathrm{n}=21)(\mathrm{p}=0,002)$ e escolaridade baixa (até $1^{\circ}$ ciclo fundamental completo) com 39,6\% $(\mathrm{n}=38)(\mathrm{p}=0,627)$. Quanto à comorbidades autorreferidas, $38,5 \%(\mathrm{n}=37)$ das mulheres incontinentes apresentaram hipertensão arterial sistêmica $(\mathrm{p}=0,96), 17,7 \% \quad(\mathrm{n}=17)$ diabetes $(\mathrm{p}=0,982)$ e $28,1 \%(\mathrm{n}=27)$ constipação intestinal $(\mathrm{p}=0,101)$ (Tabela I).

Encontrou-se que as incontinentes tiveram em média 3,9 gestações $(\mathrm{DP}=2,28)$, sendo que 79,2\% $(\mathrm{n}=76)$ tiveram até 5 filhos. Com relação ao tipo de parto, 41,7\% $(\mathrm{n}=35)$ apenas vaginal, e quanto a menopausa $53,1 \% \quad(\mathrm{n}=51)$ estavam nesse período (Tabela II).

No que se refere aos hábitos de vida, as mulheres incontinentes apresentaram maior percentual de prática de atividade física $(34,4 \%, \mathrm{n}=33)$, de tabagismo $(11,5 \%, \mathrm{n}=11)$ e de consumo de álcool $(21,9 \%, \mathrm{n}=21)$ (Figura 1).

Com relação ao perfil clínico, $52,1 \%(\mathrm{n}=50)$ referiram perder urina uma vez por semana ou menos, $75,0 \%(\mathrm{n}=72)$ relatam perder uma pequena quantidade e $85,4 \%(n=82)$ das mulheres apresentam IU de esforço (Tabela III).

Tabela II - Características obstétricas e ginecológicas de acordo com a presença ou não de incontinência urinária. Fortaleza, CE, 2014-2015.

\begin{tabular}{|c|c|c|c|c|c|c|c|}
\hline \multirow{2}{*}{ Variáveis } & \multicolumn{4}{|c|}{ COM IU } & \multicolumn{2}{|c|}{ SEM IU } & \multirow{2}{*}{ Valor de $p$} \\
\hline & Média \pm DP & $n=96$ & $\%$ & Média \pm DP & $\mathbf{n}=\mathbf{1 3 7}$ & $\%$ & \\
\hline Número de gestações & $3,94 \pm 2,287$ & & & $3,78 \pm 2,535$ & & & \\
\hline \multicolumn{8}{|l|}{ Número de filhos } \\
\hline $1-5$ & & 76 & 79,2 & & 115 & 83,9 & 0,487 \\
\hline $6-10$ & & 10 & 10,4 & & 13 & 9,5 & \\
\hline $16-20$ & & 0 & 0 & & 1 & 8,0 & \\
\hline \multicolumn{8}{|l|}{ Tipo de parto } \\
\hline Cesário & & 19 & 22,6 & & 22 & 17,5 & 0,087 \\
\hline Vaginal & & 35 & 41,7 & & 72 & 57,1 & \\
\hline Ambos os partos & & 30 & 35,7 & & 32 & 25,4 & \\
\hline \multicolumn{8}{|l|}{ Menopausa } \\
\hline $\operatorname{Sim}$ & & 51 & 53,1 & & 87 & 63,5 & 0,113 \\
\hline Não & & 45 & 46,9 & & 50 & 36,5 & \\
\hline
\end{tabular}

$\mathrm{IU}=$ incontinência urinária; $\mathrm{DP}=$ desvio-padrão.

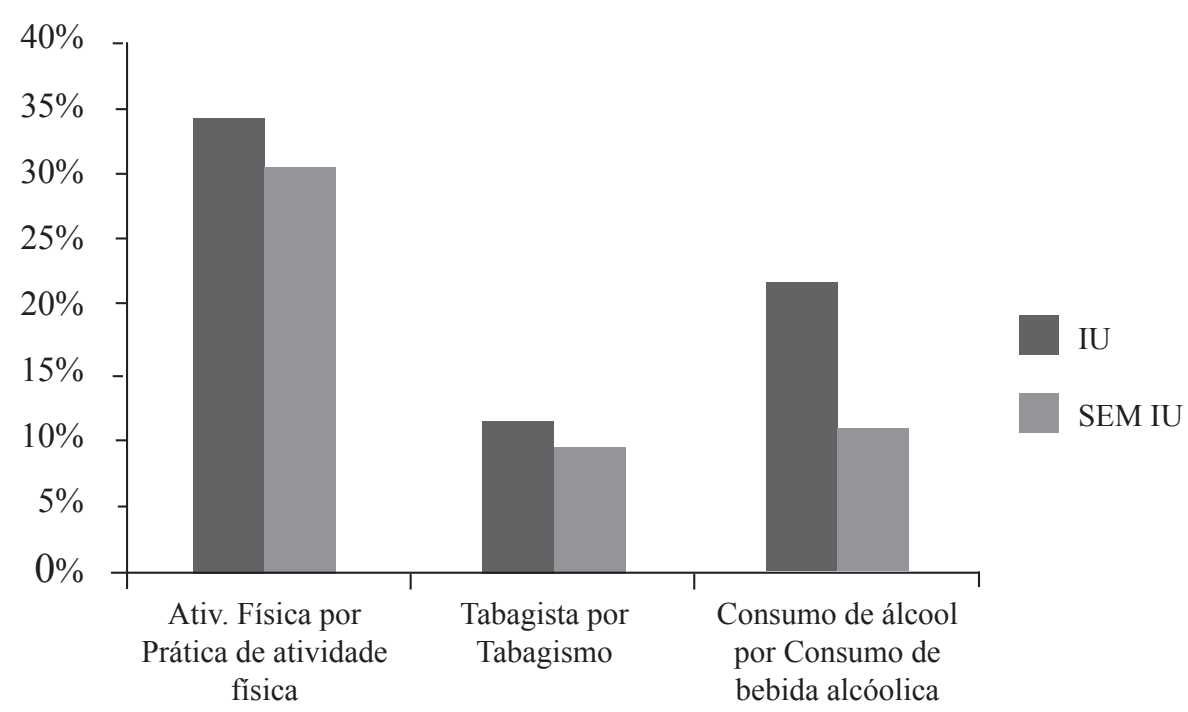

Figura 1 - Hábitos de vida de acordo com a presença ou não de incontinência urinária. Fortaleza, CE, 2014-2015.

IU = incontinência urinária 
Tabela III - Perfil urinário de mulheres com incontinência urinária. Fortaleza, CE, 2014-2015.

\begin{tabular}{|c|c|c|}
\hline Variáveis & Frequência & $\%$ \\
\hline \multicolumn{3}{|l|}{ Perda Urinária } \\
\hline uma vez por semana ou menos & 50 & 52,1 \\
\hline duas ou três vezes por semana & 22 & 22,9 \\
\hline uma vez ao dia & 5 & 5,2 \\
\hline diversas vezes ao dia & 17 & 17,7 \\
\hline o tempo todo & 2 & 2,1 \\
\hline \multicolumn{3}{|l|}{ Quantidade de urina } \\
\hline pequena quantidade & 72 & 75,0 \\
\hline moderada quantidade & 14 & 14,6 \\
\hline grande quantidade & 10 & 10,4 \\
\hline \multicolumn{3}{|l|}{ Classificação de IU } \\
\hline esforço & 82 & 85,4 \\
\hline urgência & 32 & 33,3 \\
\hline mista & 21 & 21,9 \\
\hline
\end{tabular}

IU=incontinência urinária.

\section{DISCUSSÃO}

No presente estudo, a faixa etária prevalente para perda urinária foi entre 40 a 45 anos. A idade é um dos fatores para ocorrência de IU, afetando principalmente as mulheres com faixa etária bem avançada, no período do climatério e menopausa $^{(7)}$. Esses resultados estão de acordo com outro estudo $^{(12)}$, onde a prevalência variou de acordo com a faixa etária sendo que $81 \%$ das mulheres com incontinência encontravam-se entre 40 e 59 anos.

Corroborando com o presente estudo, uma pesquisa sobre perfil sociodemográfico e clínico de usuárias da rede pública de Belo Horizonte MG, constatou que $62 \%$ das mulheres eram casadas, $19 \%$ solteiras e a maioria possuía o ensino fundamental completo ${ }^{(13)}$. O reduzido grau de instrução parece constituir fator que limita a adesão dos indivíduos aos cuidados de saúde. A falta de instrução, além de fatores sociais, culturais e econômicos, dificulta a conscientização sobre cuidados com a saúde, adesão ao tratamento adequado e a manutenção de um estilo de vida livre de exposição aos fatores de riscos ${ }^{(14)}$.

A prevalência de IU é apontada como maior mulheres brancas $^{(15)}$. Estudo epidemiológico ${ }^{(16)}$ realizado no estado de Michigan nos Estados Unidos com 2714 mulheres com idade entre 35 a 64 anos constatou maior episódio de perda urinária em brancas $(33,1 \%)$, comparadas às negras $(14,6 \%)$. Estes achados se opõem aos do presente estudo onde houve uma significância e prevalência de IU em mulheres pardas.

As alterações miccionais, provavelmente, estejam relacionadas com tratamento da Hipertensão Arterial Sistêmica, devido ao uso das medicações anti hipertensivos e diuréticos, pois aumentariam o débito urinário ${ }^{(17)}$. Porém, no presente estudo não houve associação desta variável com IU.

Os resultados encontrados na presente pesquisa revelaram não haver associação significativa entre IU e diabetes, discordando do achado na literatura ${ }^{(18)}$, onde há maior chance de mulheres com DM referir sintomas de perda urinária, devido a alterações da vascularização do assoalho pélvico-vesical, à hiperglicemia, aumentando a freqüência urinária, e mudanças nos mecanismos neurofisiológicos do sistema genito urinário ${ }^{(19)}$.

A constipação intestinal afeta a função urológica, pois o estiramento do reto comprime a bexiga, levando à retenção da urina, e consequentemente à infecção urinária, além da força feita durante a evacuação intestinal, causando lesão da musculatura pélvica( ${ }^{(7)}$. Apesar do atual estudo não ter demonstrado associação significativa da constipação com a IU, observou-se que as mulheres incontinentes apresentaram um maior percentual desse fator de risco.

Nas variáveis tipos de parto e número de gestação e filhos, observou-se na presente pesquisa que não houve associação com a IU. Contudo o tipo de parto vaginal foi o mais frequente nas mulheres incontinentes, estando de acordo com o estudo realizado em Santo André em São Paulo com 253 mulheres, afirmando que parto vaginal é um fator de risco para o desenvolvimento de alterações miccionais $^{(20)}$.

O parto vaginal é um fator de risco para ocorrência de incontinência urinária, em virtude do trauma neuromuscular do assoalho pélvico e por estiramento ou compressão mecânica dos nervos pélvicos ${ }^{(14)}$, ou ainda, pela pressão e distensão do assoalho pélvico com a passagem da cabeça do recém-nascido durante o segundo estágio do parto. 
Estudos não são conclusivos quanto à relação entre a menopausa e a perda urinária, porém, muitas mulheres relataram o surgimento da incontinência urinária neste período $^{(14)}$, corroborando com o resultado da atual pesquisa, onde a maioria das incontinentes autorreferiam estar na menopausa.

Referindo-se aos hábitos de vida, a influência do tabagismo e ingestão de álcool na IU ainda é controverso, entretanto, no que tange o tabagismo, a influência da nicotina na contração do detrusor e a ocorrência de tosse em fumantes poderiam ser uma das causas das perdas urinárias $^{(21)}$. Em outras pesquisas, no entanto, o tabagismo não apresentou associação significativa à $\mathrm{IU}^{(22)}$, mostrandose similar à presente pesquisa.

No presente estudo, a prevalência de consumo de bebida alcoólica foi no grupo de mulheres incontinentes, corroborando com outro estudo envolvendo 298 mulheres japonesas no qual mostrou maior ingestão de álcool nas mulheres com IU. Contudo, poucos estudos foram desenvolvidos comprovando a relação entre a ingestão de álcool e a incontinência urinária ${ }^{(23)}$.

Por fim, apesar dos objetivos dessa pesquisa terem sido alcançados, sugere-se que através desse estudo, outras pesquisas sejam elaboradas e que os serviços de atenção primária sejam melhor capacitados para a tomada de atitudes eficazes para prevenção e tratamento da IU, investindo na saúde e qualidade de vida das mulheres.

\section{CONCLUSÃO}

Encontrou-se elevada prevalência de incontinência urinária em mulheres climatéricas, destacando maior ocorrência nas mais jovens e de cor parda.

\section{REFERÊNCIAS}

1. Maron L, Leal A, Bandeira D, Macedo PS, Garcia SS, Silva EB. A assistência às mulheres no climatério: um estudo bibliográfico. Revista Contexto \& Saúde. 2011;10(20):545-50.

2. Mishra GD, Cardozo L, Kuh D. Menopausal transition and the risk of urinary incontinence: results from a British propective cohort. BJU Int. 2010;106(8): 1170-5.

3. Thyssen HH, Clevin L, Olesen S, Lose G. Urinary incontinence in elite female athletes and dancers. Int Urogynecol J Pelvic Floor Dysfunct. 2002;13(1):15-7.

4. Torrealba FCM, Oliveira LDR. Incontinência urinária na população feminina de idosas. Ensaios e Ciência. 2010;14(1):159-175.
5. Haylen BT, Ridder D, Freeman RM, Swift SE, Berghmans B, Lee $J$, et al. An International Urogynecological Association (IUGA)/International Continence Society (ICS) joint report on the terminology for female pelvic floor dysfunction. Int Urogynecol J Pelvic Floor Dysfunct. 2010;21(1):5-26.

6. Kruger AP, Luz SCT, Virtuoso JF. Home exercises for pelvic floor in continente womem one year after physical therapy treatment for urinary incontinence: an observational study. Braz J Phys Ther. 2011;15(5): 351-6.

7. Higa R, Lopes MHBM, Reis MJ. Fatores de risco para incontinência urinária na mulher. Rev Esc Enferm USP. 2008;1(42):187-92.

8. Imamura M, Abrams P, Bain C, Buckley B, Cardozo L, Cody, et al. Systematic review and economic modelling of the effectiveness and cost-effectiveness of non-surgical treatments for women with stress urinary incontinence. Health Technol Assess. 2010;14(40): $1-118$.

9. Sievert KD, Amend B, Toomey PA, Robinson D, Milsom I, Koelbl H, et al. Can we prevent incontinence? ICI-RS 2011. Neurourol Urodyn. 2012;31(3):390-9.

10. Sacomori C, Negri NB, Cardoso FL. Incontinência urinária em mulheres que buscam exame preventivo de câncer de colo uterino: fatores sociodemográficos e comportamentais. Cad Saúde Pública. 2013;29(6): $1251-9$.

11. Tamanini JTN, Dambros M, D'Ancona CAL, Palma PCR, Netto Jr NR. Validação para o português do "International Consultation on Incontinence Questionnaire - Short Form" (ISIQ-SF). Rev Saúde Pública. 2004;38(3):438-44.

12. Oliveira E, Takano CC, Sartori JP, Araújo MP, Pimentel SHC, Sartori MGF, et al. Trato urinário, assoalho pélvico e ciclo gravídico-puerperal. Femina. 2007;35(2):89-94.

13. Figueiredo EM, Lara JO, Cruz MC, Quintão DMG, Monteiro MVC. Perfil sociodemográfico e clínico de usuárias de Serviço de Fisioterapia Uroginecológica da rede pública. Rev Bras Fisioter.2008;12(2):136-42.

14. Berlezi EM, Dal Bem A, Antonello C, Leite MT, Bertolo EM. Incontinência urinária em mulheres no período pós-menopausa: um problema de saúde pública. Rev Bras Geriatr Gerontol. 2009;12(2):159-73.

15. Leroy LS, Lopes MHBM, Shimo AKK. A incontinência urinária em mulheres e os aspectos raciais: uma revisão de literatura. Texto \& Contexto Enferm. 2012;21(3):692-701. 
16. Fenner DE, Trowbridge ER, Patel DL, Fultz NH, Miller JM, Howard D, et al. Establishing the prevalence of incontinence study: racial differences in women's patterns of urinary incontinences. J Urol. 2008;179(4):1455-60.

17. Jardim ADI, Mazzo A, Girão FB, Sonobe HM, Souza MC. Hipertensão arterial e incontinência urinária no idoso: revisão integrativa da literatura. Rev Cuid. 2011;5(1):38-43.

18. Cavalcante KVM, Silva MIGC, Bernardo ASF, Souza DE, Lima TCGC, Magalhães AG. Prevalência e fatores associados à incontinência urinária em mulheres idosas. Rev Bras Promoç Saúde. 2014;27(2):216-23.

19. Danforth KN, Townsend MK, Curhan GC, Resnick NM, Grodstein F. Type 2 diabetes mellitus and risk of stress, urge, and mixed urinary incontinence. J Urol. 2009;181(1):193-7.

20. Oliveira E, Zuliani LMM, Ishicava J, Silva SV, Albuquerque SSR, Souza AMB, et al. Avaliação dos fatores relacionados à ocorrência da incontinência urinária feminina. Rev Assoc Med Bras. 2010;56(6):688-90.

21. Locks $\mathrm{MOH}$. Incontinência urinária na mulher idosa hospitalizada: desafios para assistência de enfermagem [tese]. Santa Catarina: Universidade Federal de Santa Catarina, Centro de Ciências da Saúde, Programa de Pós-Graduação em Enfermagem; 2013.
22. Gomes GV, Silva GD. Incontinência urinária de esforça em mulheres pertencentes ao Programa de Saúde da Família de Dourados-MS. Rev Assoc Med Bras. 2010;56(6):649-54.

23. Lee AH, Hirayama F. Alcohol consumption and female urinary incontinence; a community-based study in Japan. Int J Urol. 2012;19(2):143-8.

\section{Endereço do primeiro autor:}

Ticiana Mesquita de Oliveira

Universidade de Fortaleza - UNIFOR

Av. Washington Soares, 1321

Bairro: Edson Queiroz

CEP 60.811-905 - Fortaleza - CE - Brasil

E-mail: ticimesquita@unifor.br

\section{Endereço para correspondência:}

Ilana Nogueira Bezerra

Programa de Pós-Graduação em Saúde Coletiva

Universidade de Fortaleza - UNIFOR

Av. Washington Soares, 1321, Bloco S

Bairro: Edson Queiroz

CEP 60.811-905 - Fortaleza - CE - Brasil

E-mail: ilana.bezerra@yahoo.com.br 